\title{
Thromboprophylaxis in cancer patients in hospice
}

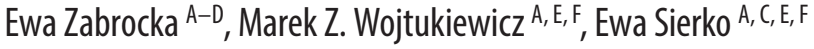 \\ Department of Oncology, Medical University of Bialystok, Poland \\ A - research concept and design; B - collection and/or assembly of data; $C$ - data analysis and interpretation; \\ $\mathrm{D}$ - writing the article; $\mathrm{E}$ - critical revision of the article; $\mathrm{F}$ - final approval of the article
}

\section{Address for correspondence \\ Ewa Sierko}

E-mail: ewa.sierko@iq.pl

Funding sources

None declared

Conflict of interest

None declared

Received on May 9, 2016

Reviewed on June 17, 2016

Accepted on August 9, 2016

DOI

$10.17219 /$ acem/64593

\section{Copyright}

Copyright by Author(s)

This is an article distributed under the terms of the

Creative Commons Attribution Non-Commercial License

(http://creativecommons.org/licenses/by-nc-nd/4.0/) 


\section{Introduction}

Venous thromboembolism (VTE) is the second most common cause of death among cancer patients. ${ }^{1}$ It consists of 2 entities: deep vein thrombosis (DVT) and pulmonary thromboembolism (PE). The relative risk of VTE is 6-fold higher in cancer patients compared to healthy control as, the meta-analyses have shown. ${ }^{2}$ Considering cancer patients in general, symptomatic DVT has been demonstrated in $15 \%$, and autopsies have revealed a $50 \%$ prevalence of PE in this group of patients. ${ }^{3}$ Regarding palliative care units, evidence of asymptomatic DVT was found in 52\% of 287 patients screened by Johnson et al. ${ }^{4}$ In another study, clinically overt VTE was reported in nearly $10 \%$ of 712 participants. ${ }^{5}$

Hypercoagulability, venous stasis and endothelial damage, known as the Virchow triad, are the main risk factors contributing to thrombosis. Each of the triad's components can be affected by cancer. Tumor elaboration of tissue factor, microparticles and inflammatory cytokines result in the activation of coagulation cascade. ${ }^{6}$ Venous stasis is caused by either vessel compression by a tumor mass or prolonged bed confinement, whereas vessel damage can occur as the consequence of e.g. chemotherapy, indwelling central venous catheters or direct tumor invasion. ${ }^{2,7}$ Of note, activated platelets, coagulation and fibrinolytic factors are involved in angiogenesis and both local and distal progression of cancer. ${ }^{8}$ In general, VTE predisposing factors in oncological patients could be divided into cancer-, treatment- and patient-dependent types (Table 1).

VTE is not only associated with a significant reduction in the overall survival of patients with malignancy, but also presents with burdensome symptoms including limb and/ or chest pain and dyspnea. In a qualitative study by Seaman et al., cancer patients being treated for VTE found the symptoms of VTE very distressful - both physically and psychologically. ${ }^{2,9}$ According to the findings of Johnson et al., $9 \%$ of likely venous thrombosis were symptomatic at the

Table 1. Factors predisposing oncological patients to venous thromboembolism

A. Cancer-dependent
advanced stage or metastatic disease
type and stage of cancer (particularly pancreatic, gastric, ovarian, lung)
B. Treatment-dependent
recent surgery
active chemotherapy (its type and duration) and radiotherapy
prothrombotic drugs: erythropoiesis stimulating factors,
bevacizumab, thalidomide, lenalidomide, tamoxifen, antiandrogens,
megestrol
central venous catheters
C. Patient-dependent
advanced age
race (higher risk in African Americans)
obesity
comorbidities, e.g. renal, liver or pulmonary disease
prolonged immobilization
prior history of thrombosis

time of original diagnosis, but another $32 \%$ of cancer patients with likely DVT subsequently developed symptoms which included significant lower limb pain and swelling. ${ }^{4}$

A randomized control trial has demonstrated that primary thromboprophylaxis (TPX) can significantly reduce the incidence of VTE in immobilized cancer patients. ${ }^{10}$ The data focusing on palliative care patients, however, is unsatisfactory. Weber et al. performed a prospective randomized study to determine the utility of prophylactic anti-coagulation in cancer patients hospitalized in a palliative care unit with an estimated life expectancy of 6 months or less. ${ }^{11}$ Although neither harm nor an unequivocal benefit from the TPX was revealed, the results should be verified on a larger study group since only 20 patients were enrolled in the study. ${ }^{11}$ Another study showed that patients without primary TPX on admission to hospice were at higher risk of developing VTE symptoms compared to patients receiving primary TPX. ${ }^{12}$ However, the difference was not statistically significant and the proportion of patients receiving primary TPX ( $4 \%$ only) was probably too small to derive a firm conclusion in this matter.

Although routinely used in patients with advanced malignancy in oncology wards, primary TPX is relatively rarely administered in the palliative care setting, including hospice. Contrary to the formal and unequivocal guidelines for management of pain, vomiting and nausea, nutrition or sedation at the end of life, no unanimous evidence-based guidance on VTE prophylaxis and management in patients approaching death is available. This largely results from the lack of sufficiently powered studies concerning the factual symptom burden of VTE in this group of patients and its impact on QoL. In the absence of such data the relevance of TPX in terminal care remains vague.

Moreover, the problem is controversial in terms of ethics. According to the definition of palliative care established by the World Health Organization, dying is recognized as a natural process and the main target of palliation is not to postpone death, but to improve QoL through the alleviation of distressing symptoms. ${ }^{13}$ It is not clearly defined whether TPX prolongs life in patients approaching death, but neither has it been investigated if and to what extent anticoagulation could relieve the burdensome symptoms associated with VTE at the terminal stage of malignancy.

Nowadays, in the era of highly developed treatment modalities and advanced supportive care, the significance of hospice not solely as a place of dying, but also as a measure of the improvement of patient condition, seems to become more and more pronounced. On average, approx. 1 in 5 hospice patients were discharged alive from hospices in the USA in 2010. ${ }^{14}$ Therefore, although the interventions to prevent long-term complications of malignancies are frequently perceived futile and unnecessary in a hospice setting, the problem should not be neglected.

In our paper, we review the data on both physicians' and cancer patients' attitudes toward anticoagulation at the end of life, analyze available methods of thromboprophylaxis 
in the view of this group of patients and review the research and recommendations of international cancer societies concerning primary TPX in terminal stage cancer patients.

\section{Attitudes of physicians and patients to primary thromboprophylaxis in hospice}

Physicians working in palliative medicine have been found to significantly underestimate the likely prevalence of VTE in hospice inpatients. One study has shown they estimated the risk to be $1-5 \%$ only, whereas the actual prevalence is most likely at least 5 -fold higher. ${ }^{4,15}$ The conviction of a low prevalence of VTE led medical directors of palliative care units interviewed by Noble et al. not to consider symptomatic VTE a clinical priority. ${ }^{16}$ Moreover, some of them expressed the view that fatal PE is a less distressing mode of death ('quick and easy') compared to other cancerrelated pathologies patients may experience, should they live longer. Simultaneously, TPX was considered not in line with the philosophy of palliative care as it was regarded as a life-prolonging intervention. Finally, TPX was perceived not to be a cost-effective intervention since it did not alter the outcome at this stage of the disease. ${ }^{16}$

However, physicians' approach to VTE prevention has been shown to evolve. There has been a shift from avoiding the use of low molecular weight heparin (LMWH) to its more common implementation in palliative care units. Among palliative care specialists surveyed by Noble et al. in 2000, 62\% routinely stopped TPX, whereas in 2005 it was only $18 \% .{ }^{17}$ Likewise, a more individual approach is observed, which takes into consideration patients' own preferences regarding anticoagulation.

Decisions on limitation of treatment at the end-oflife period pose ethical challenges and are perceived by physicians as difficult. ${ }^{18}$ It is still vague when, if at all, TPX should be ceased in patients approaching death. In a survey study in which experts in palliative care, oncology, intensive care and anticoagulation were enrolled, all the surveyed physicians opted to withdraw or withhold primary prophylaxis in patients with a Karnofsky Performance Status (KPS) of less than 10, defined as "moribund". ${ }^{19}$ Whereas one quarter of doctors would have employed prophylactic anticoagulants when KPS was at least 20, most physicians (85\%) declared they would have implemented primary TPX if KPS was over 40. There was an inverse relation between patient's performance status and the readiness of physicians to prescribe TPX. The study has also shown that doctors' decisions of whether to employ VTE prevention were made with a potential risk-benefit assessment.

Studies have revealed that palliative care inpatients wish to be involved in the decision-making process, particularly concerning withdrawal or non-administration of TPX. ${ }^{20,21}$
Moreover, they expressed their concern about potential disqualification from TPX due to the advanced malignancy they were struggling with. In the study by Gartner et al., $87 \%$ of patients who had been involved in the decision process opted to receive TPX. ${ }^{21}$

Anticoagulation is often thought to be painful and bothersome for hospice patients due to the need for daily subcutaneous injections associated with the use of low molecular weight heparin (LMWH), one of the most commonly implemented anticoagulants in routine TPX, which could decrease QoL. ${ }^{8}$ However, in one study, all of the 28 patients interviewed receiving palliative care, who had been given LMWH for at least 5 consecutive days, found LMWH an acceptable intervention, and many said it improved their QoL by giving them a feeling of safety and reassurance. ${ }^{20}$ They perceived optimizing QoL as not only treating symptoms but also taking measures to prevent other symptoms. Another study on the acceptability of long-term LMWH use revealed that for terminally ill patients it was important to know that something active was being done despite the hopelessness of their clinical situation. ${ }^{22}$

LMWH was also found acceptable by palliative care patients receiving TPX in a long-term setting for the treatment of VTE. ${ }^{9,2}$

Although the aforementioned studies have demonstrated a generally positive impact of TPX on QoL, it is particularly difficult to draw generalized conclusions from studies concerning VTE prevention performed on patients under palliative care due to the often various performance statuses of the participants and the lack of a uniform tool for QoL assessment.

\section{Selection of thromboprophylaxis method with reference to hospice practice}

There are various methods of thromboprophylaxis widely used in clinical practice. Patients approaching death, however, constitute a particular subgroup of patients in whom not every method might be suitable.

The most popular forms of mechanical TPX include compression stockings and intermittent pneumatic compression (IPC). Despite the fact that it does not cause hemorrhagic complications, IPC has been poorly studied in non-surgical cancer patients. Also, little research on the use of elastic stockings is available in the literature concerning patients under palliative care. Nevertheless, the available data has shown the stockings were found uncomfortable and unacceptable by patients, causing itching, sweating and an unpleasant feeling of pressure, and therefore decreasing QoL. ${ }^{10}$ Oral vitamin K antagonists (VKA) inhibit the synthesis of vitamin K-dependent coagulation factors. VKA have numerous interactions with food and drugs used in supportive or active oncological treatment. 
They also have a narrow therapeutic window, therefore the treatment requires titration and further monitoring based on international normalized ratio (INR). Repeated blood sampling is burdensome for patients and may decrease adherence to the treatment. ${ }^{8}$ Additionally, patients under palliative or hospice care have been shown to require more frequent INR control due to difficulties in achieving and maintaining therapeutic drug levels. ${ }^{23}$ This mainly results from, e.g., malnutrition, diarrhea, emesis or liver failure, common in patients with advanced malignancy. As a matter of fact, cancer patients who are treated with VKA for VTE have a substantial rate of recurrent VTE. ${ }^{23}$ VKA is also found less effective than low molecular weight heparin in patients with cancer-associated thrombosis. ${ }^{23}$ However, it has been not established whether the conclusion of these findings could be extrapolated to patients receiving primary TPX, particularly in a hospice setting.

Unfractionated heparin (UFH), which is factor Xa and thrombin inhibitor, can be administered either intravenously or subcutaneously, has a good safety profile in terms of renal insufficiency and its effects can be reversed by protamine sulfate. UFH, however, should be given more than once daily, requires monitoring of activated partial thromboplastin time (APTT) and can cause heparin-induced thrombocytopenia (HIT). Therefore, it is used in selected patients only, especially those with significant impairment of renal function.

The new generation of heparin, low molecular weight heparin (LMWH), is currently more often implemented than UFH in TPX. Compared to UFH, it has a longer halflife and can be administered only once a day, is characterized by higher bio-availability and entails much lower risk of HIT. ${ }^{24}$ In comparison with warfarin, LMWH is more effective in the prevention of recurrent VTE in cancer patients and safer with respect to bleeding complications. ${ }^{25}$ In the American College of Chest Physicians (CHEST) Guidelines, LMWH is suggested as the preferred long-term treatment for VTE in cancer patients. ${ }^{23}$ Routine monitoring of coagulation parameters is not required in most cases, although there is a need for repeated subcutaneous injections, which could adversely impact QoL. However, as mentioned above, it was shown to be a trivial intervention, acceptable by palliative care inpatients, with little or no impact on QoL and bruising as the only negative experience reported. ${ }^{20}$ Although LMWH has been reported to increase the overall survival in cancer patients, the survival benefit was not statistically significant in the subgroup of patients in the advanced stages of malignancy. ${ }^{26}$ Additionally, the Fragmin Advanced Malignancy Outcome Study (FAMOUS) revealed that LMWH administration did not improve 1-year survival in the final 3 months of life of advanced cancer patients. ${ }^{27}$ Life prolongation, however, is not in line with the principles of end-of-life care and should not be considered important in a hospice setting.
Renal insufficiency is one of the contraindications to LMWH use. Given that this condition can be found in more than half of cancer patients, LMWH utilization in the prevention of VTE in this group of patients might be challenging and requires dose adjustment (with the exception of tinzaparin, which does not accumulate in patients with renal function impairment and can be administered without dose corrections). ${ }^{28}$

Fondaparinux is an indirect inhibitor of factor Xa. It was demonstrated to be an option for VTE prevention in cancer patients hospitalized for acute medical illness or surgery. ${ }^{29}$ On account of the lack of a reversal agent and significant dependence on renal clearance, its employment in the palliative care setting might be limited, although there are reports on the successful use of fondaparinux in both primary and secondary VTE prevention in oncological patients. $^{30}$

Novel oral anticoagulants (NOA) are specific inhibitors of activated factor X (apixaban, rivaroxaban and edoxaban) or thrombin (dabigatran). Less interaction with food and drugs, oral administration and no need for drug-level monitoring make its use convenient. Until recently, no antidote was available to any of the NOA. The introduction of both idarucizumab, a humanized monoclonal antibody against dabigatran, and andexanet alfa, a molecule reversing Xa inhibitor activity, was a breakthrough in this matter. ${ }^{31,32}$

The MAGELLAN (Multicenter, Randomized, Parallel Group Efficacy and Safety Study for the Prevention of Venous Thromboembolism in Hospitalized Acutely Ill Medical Patients Comparing Rivaroxaban with Enoxaparin) study, investigating the efficacy and safety of rivaroxaban, revealed a non-significant trend of lower efficacy of rivaroxaban compared to enoxaparin in the subgroup of cancer patients as well as significantly higher bleeding risk associated with rivaroxaban over enoxaparin administration. ${ }^{8}$ Apixaban, on the other hand, was shown as effective as enoxaparin in primary TPX in the ADOPT (Apixaban dosing to optimize protection from thrombosis) trial, although related with significantly more relevant bleeding events. ${ }^{33}$ In the latest CHEST guidelines, the risk reduction for recurrent VTE has not been compared between NOA and LMWH, however, indirect comparisons make it possible to assume that LMWH may be more effective than NOA in cancer patients with VTE. ${ }^{23}$ Simultaneously, the risk reduction for recurrent VTE is similar between NOA and VKA, therefore VKA is no longer suggested over NOA in cancer patients treated for VTE. ${ }^{23}$ Although NOA is an attractive option for TPX in oncological patients, the clinical trials designed exclusively for cancer patients - not to mention palliative care patients - are lacking. 


\section{Thromboprophylaxis in end-of-life care: Current guidelines and challenges}

The National Institute for Health and Clinical Excellence (NICE) recommends the use of pharmacological VTE prophylaxis in palliative care for patients who have potentially reversible acute pathology. ${ }^{34}$ The potential risks and benefits as well as the opinion of patients and their families should be taken into account. NICE recommends the use of fondaparinux, LMWH or UFH (for patients with renal failure). Patients admitted for terminal care or those commenced on an end-of-life care pathway should not be considered for routine pharmacological or mechanical VTE prophylaxis. Additionally, the decisions regarding TPX for patients in palliative care should be reviewed daily.

Previously, VTE prevention was supported by the American College of Chest Physicians (CHEST) in the palliative care setting in selected patients only, i.e. in whom it could be expected that TPX could prevent progressive deterioration of QoL. ${ }^{35}$ However, the current guidelines of the CHEST on VTE prevention do not refer to the population of palliative care patients. ${ }^{36}$ Prophylactic-dose LMWH or low-dose UFH is recommended in outpatients with solid tumors and additional risk factors for VTE (e.g. immobilization), provided they are at low risk of bleeding. On the other hand, routine use of TPX is not supported by the CHEST in chronically immobilized patients residing at nursing homes. ${ }^{36}$

According to National Comprehensive Cancer Network (NCCN) guidelines, no routine TPX is recommended outside of clinical trials in cancer patients after being discharged from the hospital nor in ambulatory cancer patients remaining at risk of VTE (e.g. advanced stages of cancer, poor performance status, medical comorbidities). ${ }^{37} \mathrm{TPX}$ use in the palliative care setting is not referred to in the guidelines.

Anticoagulation should not be used to extend the survival of cancer patients without VTE in the absence of other indications, according to American Society of Clinical
Oncology (ASCO) recommendations. ${ }^{10,38}$ Routine TPX can be considered only in selected high-risk outpatients with cancer, however the application of this recommendation to palliative or end-of-life care was not specified. ${ }^{38}$

The European Society for Medical Oncology (ESMO), despite recommending prophylaxis with UFH, LMWH or fondaparinux in hospitalized, bedridden cancer patients with an acute medical complication, has not referred to the problem of VTE prevention in palliative care. ${ }^{39}$ The aforementioned guidelines are summarized in Table 2 .

Since the decisions on whether to implement TPX in palliative care are often left to physicians' individual assessment, in recent years it seems to be more common for specialist palliative care units to have their own policy regarding VTE prevention. Among palliative care units in Great Britain, only $3 \%$ had a TPX policy in 2000, whereas in 2005 the number had increased to $7 \% .{ }^{17}$

Given that evidence based on large studies is still lacking, doctors are compelled to rely on their own experience in everyday practice. The studies concerning actual symptom burden associated with VTE in end-of-life care and its cumulative effect on QoL are pending, as is research on the extent to which TPX could reduce VTE symptom burden and improve QoL. Certainly, not the survival rates but the quality of the remaining lifetime should be the most important measure of outcome in the research regarding hospice patients. For that reason, the results of studies involving hospitalized cancer patients in general are not necessarily applicable to the hospice setting. Unfortunately, a reliable tool for QoL assessment is still lacking.

The use of anticoagulants in hospice patients should be also verified in terms of bleeding, since the population of advanced cancer patients is frequently at hemorrhagic risk due to renal and liver failure, malnutrition or the metastatic process involving organs participating in hemostasis (liver, bone marrow). In one study on TPX in palliative care units, contraindications for TPX were present in 25\% of all cancer patients and in $35 \%$ of the bedridden ones. ${ }^{21}$ The rate was even higher in the study by Johnson et al., where primary TPX was contraindicated in $42.6 \%$ out of 1164 hospice patients. ${ }^{12}$

Table 2. Summary of guidelines for thromboprophylaxis in the palliative care setting

\begin{tabular}{|c|c|c|}
\hline Recommendation & Author & References \\
\hline $\begin{array}{l}\text { TPX not recommended at the end-of-life care pathway. May be considered in } \\
\text { patients admitted with reversible acute pathology }\end{array}$ & $\begin{array}{l}\text { National Institute for Health and Clinical } \\
\text { Excellence (NICE) }\end{array}$ & 34 \\
\hline $\begin{array}{l}\text { No guidelines on TPX in palliative care. TPX recommended in immobilized } \\
\text { outpatients with solid tumors, but opposed in immobilized patients at nursing } \\
\text { homes }\end{array}$ & $\begin{array}{l}\text { American College of Chest Physicians } \\
\text { (CHEST) }\end{array}$ & 36 \\
\hline $\begin{array}{l}\text { No guidelines on TPX in palliative care. Routine TPX use should be limited to clinical } \\
\text { trials only }\end{array}$ & $\begin{array}{l}\text { National Comprehensive Cancer Network } \\
\text { (NCCN) }\end{array}$ & 37 \\
\hline $\begin{array}{l}\text { No guidelines on TPX in palliative care. TPX should not be the life-prolonging } \\
\text { procedure. Can be considered in selected high-risk cancer outpatients }\end{array}$ & $\begin{array}{l}\text { American Society of Clinical Oncology } \\
\qquad(\mathrm{ASCO})\end{array}$ & 10,38 \\
\hline No guidelines on TPX in palliative care setting & $\begin{array}{l}\text { European Society for Medical Oncology } \\
\qquad(\text { ESMO) }\end{array}$ & 39 \\
\hline
\end{tabular}


The economic aspect of TPX in hospice should also be considered. The costs of drugs and nursing time may be considerable, and for some hospice, mainly financed with donations, this may be a barrier difficult to overcome. Chambers calculated that the drug costs of one particular hospice would increase by $28 \%$ if LMWH were administered to all immobile cancer patients. ${ }^{40}$ Hopefully, the use of generic versions of LMWH would be a less expensive alternative. Moreover, as calculated, LMWH should be administered to 190 patients with advanced cancer to prevent one symptomatic VTE. Statistically, preventing less than one episode of VTE annually would be accompanied by 3.5 additional bleeding complications. ${ }^{40}$ The cost-effectiveness calculations are particularly challenging in the population of hospice patients since an increased risk of VTE is often accompanied by high risk of bleeding events. Since the treatment of hemorrhagic episodes generates additional costs, this should be also taken into account.

Finally, the vast majority of research and recommendations focus on palliative care as a whole, without the distinction of a hospice setting. Palliative care units provide care to a wider group of patients, including those who are not dying. In other words, palliative care is not limited to end-of-life care and can be applied in any stage of the disease, in contrast to hospice. A hospice patient and palliative patient may significantly differ in performance status and life expectancy, therefore it would be reasonable for the authors of future studies and guidelines to refer to these 2 groups of patients rather separately.

\section{Conclusions}

As long as there is no clear evidence from large, randomized studies supporting the use of thromboprophylaxis in hospice, it seems that TPX in this group of patients should not be a routine practice. Patients ought to be involved in the decision-making process concerning TPX. Studies on the relations between VTE symptom burden, TPX and QoL should be performed on a hospice population, not palliative in general.

\section{References}

1. Pruemer J. Prevalence, causes, and impact of cancer-associated thrombosis. Am J Health Syst Pharm. 2005;62(22 Suppl 5):S4-S6.

2. Cunningham MS, White $B, O^{\prime}$ Donnell J. Prevention and management of venous thromboembolism in people with cancer: A review of the evidence. Clin Oncol (R Coll Radiol). 2006;18:145-151.

3. Ambrus JL, Ambrus CM, Mink IB, Pickren JW. Causes of death in cancer patients. J Med. 1975;6:61-64.

4. Johnson MJ, Sproule MW, Paul J. The prevalence and associated variables of deep venous thrombosis in patients with advanced cancer. Clin Oncol (R Coll Radiol). 1999;11:105-110.

5. Soto-Cárdenas MJ, Pelayo-García G, Rodríguez-Camacho A, Segura-Fernández E, Mogollo-Galván A, Giron-Gonzalez JA. Venous thromboembolism in patients with advanced cancer under palliative care: Additional risk factors, primary/secondary prophylaxis and complications observed under normal clinical practice. Palliat Med. 2008; 22:965-968.
6. Falanga A, Panova-Noeva M, Russo L. Procoagulant mechanisms in tumour cells. Best Pract Res Clin Haematol. 2009;22:49-60.

7. Davis MP. Hematology in palliative medicine. Am J Hosp Palliat Care. 2004;21:445-454.

8. Gerotziafas GT, Mahé I, Elalamy I. New orally active anticoagulant agents for the prevention and treatment of venous thromboembolism in cancer patients. Ther Clin Risk Manag. 2014;10:423-436.

9. Seaman S, Nelson A, Noble S. Cancer-associated thrombosis, lowmolecular-weight heparin, and the patient experience: A qualitative study. Patient Prefer Adherence. 2014;8:453-461.

10. Lyman GH, Khorana AA, Falanga A, et al. American Society of Clinical Oncology guideline: Recommendations for venous thromboembolism prophylaxis and treatment in patients with cancer. J Clin Oncol. 2007;25:5490-5505.

11. Weber C, Merminod T, Herrmann FR, Zulian GB. Prophylactic anticoagulation in cancer palliative care: A prospective randomised study. Support Care Cancer. 2008;16:847-852.

12. Johnson MJ, McMillan B, Fairhurst C, et al. Primary thromboprophylaxis in hospices: The association between risk of venous thromboembolism and development of symptoms. J Pain Symptom Manage. 2014;48:56-64.

13. Definition of palliative care. World Health Organization Web site. www. who.int/cancer/palliative/definition/en. Accessed January 05, 2016.

14. Teno JM, Plotzke M, Gozalo P, Mor V. A national study of live discharges from hospice. J Palliat Med. 2014;17:1121-1127.

15. Johnson MJ, Sherry K. How do palliative physicians manage venous thromboembolism? Palliat Med. 1997;11:462-468.

16. Noble SI, Nelson A, Finlay IG. Factors influencing hospice thromboprophylaxis policy: A qualitative study. Palliat Med. 2008;22:808-813.

17. Noble SI, Finlay IG. Have palliative care teams' attitudes toward venous thromboembolism changed? A survey of thromboprophylaxis practice across British specialist palliative care units in the years 2000 and 2005. J Pain Symptom Manage. 2006;32:38-43.

18. Schildmann J, Hoetzel J, Baumann A, Mueller-Busch C, Vollmann J. Limitation of treatment at the end of life: An empirical-ethical analysis regarding the practices of physician members of the German Society for Palliative Medicine. J Med Ethics. 2011;37:327-332.

19. Kierner KA, Gartner V, Schwarz M, Watzke HH. Use of thromboprophylaxis in palliative care patients: A survey among experts in palliative care, oncology, intensive care, and anticoagulation. Am J Hosp Palliat Care. 2008;25:127-131.

20. Noble SI, Nelson A, Turner C, Finlay IG. Acceptability of low molecular weight heparin thromboprophylaxis for inpatients receiving palliative care: Qualitative study. BMJ. 2006; 332:577-580.

21. Gartner V, Kierner KA, Namjesky A, et al. Thromboprophylaxis in patients receiving inpatient palliative care: A survey of present practice in Austria. Support Care Cancer. 2012;20:2183-2187.

22. Noble SI, Finlay IG. Is long-term low-molecular-weight heparin acceptable to palliative care patients in the treatment of cancer related venous thromboembolism? A qualitative study. Palliat Med. 2005;19:197-201.

23. Kearon C, AkI EA, Ornelas J, et al. Antithrombotic therapy for VTE disease: CHEST guideline and expert panel report. Chest. 2016;149:315-352.

24. Martel N, Lee J, Wells PS. Risk for heparin-induced thrombocytopenia with unfractionated and low-molecular-weight heparin thromboprophylaxis: A meta-analysis. Blood. 2005;106:2710-2715.

25. Lee AY, Levine MN, Baker RI, et al. Low-molecular-weight heparin versus a coumarin for the prevention of recurrent venous thromboembolism in patients with cancer. N Engl J Med. 2003;349:146-153.

26. AklEA, van Doormaal FF, Barba $M$, et al. Parenteral anticoagulation for prolonging survival in patients with cancer who have no other indication for anticoagulation. Cochrane Database Syst Rev. 2007;CD006652.

27. Kakkar AK, Levine MN, Kadziola Z, et al. Low molecular weight heparin, therapy with dalteparin, and survival in advanced cancer: The fragmin advanced malignancy outcome study (FAMOUS). J Clin Oncol. 2004;22:1944-1948.

28. Launay-Vacher V, Oudard S, Janus N, et al. Prevalence of renal insufficiency in cancer patients and implications for anticancer drug management: The renal insufficiency and anticancer medications (IRMA) study. Cancer. 2007;110:1376-1384. 
29. Zalpour A, Kroll MH, Afshar-Kharghan V, Yusuf SW, Escalante C. Role of factor Xa inhibitors in cancer-associated thrombosis: Any new data? Adv Hematol. 2011;2011:196135.

30. Nagler M, Haslauer M, Wuillemin WA. Fondaparinux - data on efficacy and safety in special situations. Thromb Res. 2012;129:407-417.

31. Pollack CV Jr, Reilly PA, Eikelboom J, et al. Idarucizumab for dabigatran reversal. N Engl J Med. 2015;373:511-520.

32. Siegal DM, Curnutte JT, Connolly SJ, et al. Andexanet alfa for the reversal of factor Xa inhibitor activity. N Engl J Med. 2015;373: 2413-2424.

33. Goldhaber SZ, Leizorovicz A, Kakkar AK, et al. Apixaban versus enoxaparin for thromboprophylaxis in medically ill patients. N Engl J Med. 2011;365:2167-2177.

34. National Clinical Guideline Centre for Acute and Chronic Conditions. Venous thromboembolism in adults admitted to hospital: reducing the risk. London (UK): National Institute for Health and Care Excellence (NICE); 2015 Jun. 62 p. (Clinical guideline; no. 92). http://www. guideline.gov/content.aspx?id=49437. Accessed January 05, 2016.

35. Geerts WH, Bergqvist D, Pineo GF, et al. Prevention of venous thromboembolism: American College of Chest Physicians Evidence-Based Clinical Practice Guidelines ( $8^{\text {th }}$ edition). Chest 2008;133:3815-453S.

36. Kahn SR, Lim W, Dunn AS, et al. Prevention of VTE in nonsurgical patients: Antithrombotic Therapy and Prevention of Thrombosis, $9^{\text {th }}$ ed: American College of Chest Physicians Evidence-Based Clinical Practice Guidelines. Chest. 2012;141(2 Suppl), e195S-226S.

37. Clinical practice guidelines in oncology: Cancer-associated venous thromboembolic disease, v.1.2015. National Comprehensive Cancer Network Web site. www.nccn.org/professionals/physician_gls/pdf/ vte.pdf. Accessed January 05, 2016.

38. Lyman GH, Bohlke $\mathrm{K}$, Khorana AA, et al. Venous thromboembolism prophylaxis and treatment in patients with cancer: American Society of Clinical Oncology clinical practice guideline update 2014. J Clin Oncol. 2015;33:654-656.

39. Mandalà M, Falanga $A$, Roila F. Management of venous thromboembolism (VTE) in cancer patients: ESMO Clinical Practice Guidelines. Ann Oncol. 2011;22 (6 Suppl):vi85-92.

40. Chambers JC. Prophylactic heparin in palliative care: ...to a challenging idea. BMJ. 2006;332:729. 